\title{
Radioactive Ion beams in Brasil (RIBRAS) *
}

\author{
R. Lichtenthäler, A. Lépine-Szily, V. Guimarães, G.F. Lima, and M.S. Hussein \\ Departamento de Física Nuclear \\ Instituto de Física da Universidade de São Paulo \\ CP 66318, 05315-970 São Paulo SP, Brasil
}

Received on 30 October, 2002

\begin{abstract}
The study of the so called exotic nuclei constitutes an important field of research in nuclear physics. The possibility of producing secondary beams of unstable nuclei(RIB) allows the investigation of nuclei at extreme conditions as low binding energies, high angular momentum and isospin. More laboratories are going through the process of re-direction of their experimental effort towards this activity. The nuclear physics community in Brazil has enthusiastically decided to join this endeavor to provide the Pelletron/LINAC complex with two superconducting solenoids which will permit the production of secondary beams of radioactive nuclei. A description of this facility and project RIBRAS follows.
\end{abstract}

\section{The RIBRAS project}

The Pelletron-LINAC Laboratory of the University os São Pulo is working on the installation of the first of the first South America Radioactive Ion beam device (RIBRAS) $[1,3]$. This facility extends the capabilities of the original Pelletron accelerator by producing secondary beams of unstable nuclei. A drawing of this system is shown in Figure 1. The most important components in this system are the two new super-conducting solenoids.

The solenoids have $6.5 \mathrm{~T}$ maximum central field (5 T.m axial field integral) and a $30 \mathrm{~cm}$ clear warm bore, which corresponds to an angular acceptance in the range $(2 \leq$ $\theta \leq 15 \mathrm{deg})$. The system was conceived by Cryomagnetics INC and it was designed to operate following the Linac post-accelerator of maximum energy of $10 \mathrm{MeV}$.A presently under installation. This energy will be about 3 times larger than the maximum energy of the present Pelletron Tandem of $8 \mathrm{MV}$ terminal voltage (3-5MeV.A).

The liquid He consumption of these solenoids is about $0.14 \mathrm{l} / \mathrm{hr}$ in persistent mode, and $0.7 \mathrm{l} / \mathrm{hr}$ with an external current of $90 \mathrm{~A}$ at maximum magnetic field. Both of these are compatible with the capability of the He liquifier installed at Pelletron-LINAC Laboratory. The presence of the two magnets in the design is very important. The first solenoid makes an in-flight selection of the reaction products emerging from the primary target in the forward angle region. As the first magnet transmits all ions with the same magnetic rigidity $m E / Q^{2}$ the radioactive secondary beam can be rather poor. With two solenoids, it is possible to use differential energy loss in an energy degrader foil, located at the crossover point between the magnets. This degrader foil will allow the second solenoid to select the ion of interest by moving the contaminant ions out of its bandpass. Time of flight technique using pulsed primary beam is also very useful in order to identify nuclei of interest in the secondary beam. The buncher system to be used in the Pelletron accelerator to pulse the beam is presently being installed.

An additional future possibility of the two solenoid system is the production of tertiary beams using a secondary target in the middle scattering chamber. The second solenoid can be tuned to select a different magnetic rigidity producing low intensity $(1-100 / \mathrm{s})$ tertiary beams like ${ }^{9} \mathrm{Li}$, ${ }^{8} \mathrm{He}$.[3] This is in principle possible with secondary beams of $10^{7} / \mathrm{s}$ and assuming a tipically conversion efficiency of $10^{-} 5$ for the secondary reaction. In order to achieve higher secondary beam intensities one possibility for the future is the installation of an ECR ion source before the LINAC.

\section{The Physics with RIBRAS}

The two solenoids are presently installed in the 45B Pelletron beam line. It should be noted that setting up the solenoid system prior to the completion of the LINAC postaccelerator is an important consideration. This will make possible to begin experiments with a facility that is in principle similar to the TWINSOL at Notre Dame University [2]. In this first stage with the Pelletron primary beam, the Pelletron primary beam of ${ }^{7,6} \mathrm{Li}$ of 3-5 MeV.A and 1-2 $\mu \mathrm{Ae}$, will permit the production of secondary beams such as ${ }^{7} \mathrm{Be}$, ${ }^{8} \mathrm{~B},{ }^{8} \mathrm{Li},{ }^{6} \mathrm{He}$ of intensities around $10^{4}$ to $10^{6}$ particles per second. With these intensities one can perform measurements of elastic scattering angular distributions and studies of the interaction potential of systems involving exotic projectiles allowing the investigation of phenomena such as proton and neutron halo in nuclei. The possibility of studies of the scattering of secondary beams of nuclei in isomeric excited states is also a new interesting subject.

\footnotetext{
*Auxílio Pesquisa FAPESP no.97/9956-5, Projeto Temático FAPESP no.2001/06676-9, PRONEX
} 


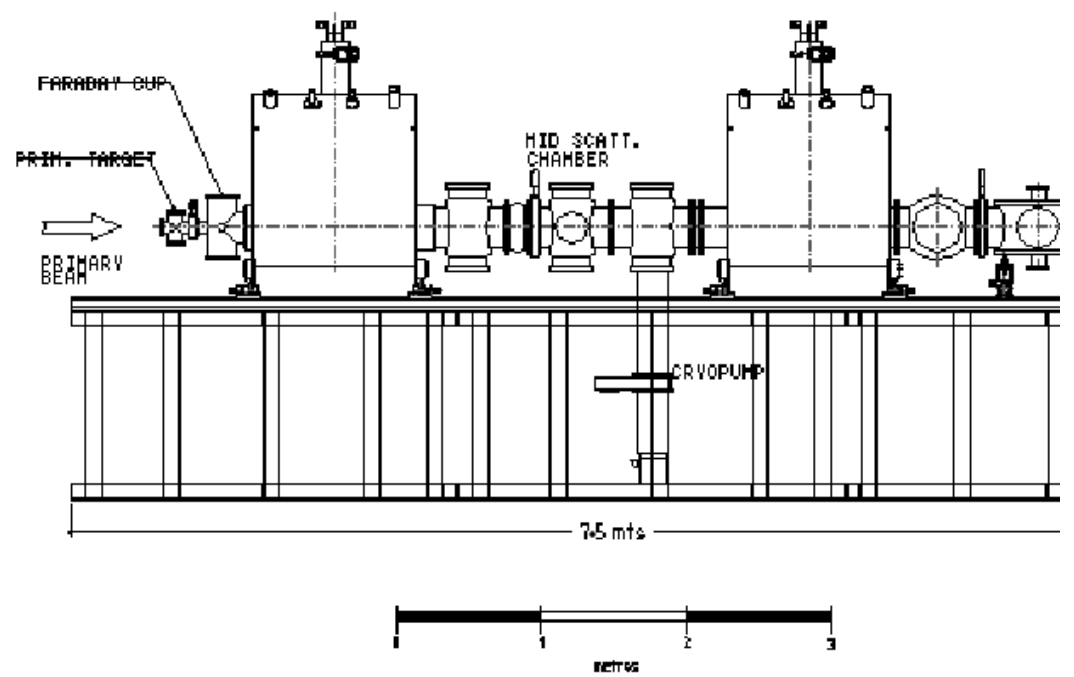

Figure 1. Scale illustration of the São Paulo - double solenoid ion-optical RIB system, RIBRAS.

Another interesting problem that can be investigated is the fusion involving nuclei near to the drip lines. It is expected that neutron and proton halo projetiles would have different behaviors in the fusion process with heavy targets below the Coulomb barrier due to the different characteristics of the halo. Measurements of fusion cross-sections involving such exotic projectiles will be in principle possible in the first stage of RIBRAS.

Probably the most important impact of the research with RIB is in nuclear astrophysics [4]. The possibility of measuring the cross-sections of capture reactions of astrophysical interest involving exotic nuclei will certainly have important consequences in the models of the primordial as well as in the explosive nucleosynthesis. Measurements of capture reactions near to the proton and neutron drip lines are of crucial importance in the rp-process and r-process responsible for the nucleosynthesis of heavy elements.

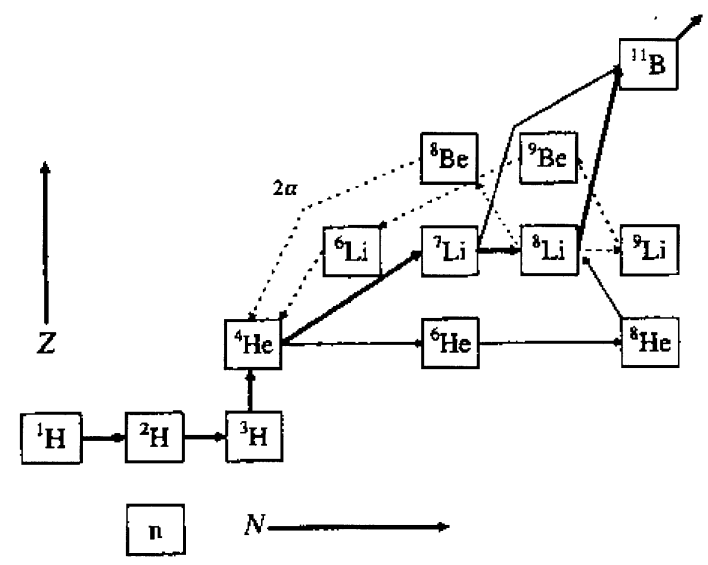

Figure 2. The reaction chain to ${ }^{11} B$ in the primordial nucleosynthesis, ref.5.

On the other hand, the primordial nucleosynthesis in- volves reactions with light nuclei that would be accessible with RIBRAS beams. In Fig. 2 is shown the reaction chain to ${ }^{11} B$ in the inhomegenous model of primordial nucleosynthesis [5]. There are new paths near to the neutron drip line involving nuclei like ${ }^{8} \mathrm{Li},{ }^{6} \mathrm{He}$ that would lead to ${ }^{11} \mathrm{~B}$. The group of researchers responsible for the installation of the two solenoid system is mainly interested in elastic scattering and fusion measurements with exotic beams and in transfer reactions of astrophysical interest.

The proposed RIBRAS facility will have several important advantages provided by the linear postaccelerator(LINAC). In particular, higher-energy (up to 10 $\mathrm{MeV} /$ nucleon), higher mass (perhaps up to $\mathrm{A}=50$ ) radioactive ion beams can be produced with beam purities approaching $80 \%$ in many cases. In addition, the pulsed time structure of the beam will provide a time-of-flight parameter that can be used to reduce backgrounds in many experiments. On a more speculative note, if Uranium beams could be accelerated to energies of a few $\mathrm{MeV}$ per nucleon, transfer induced fission reactions could be used to produce a wide variety of very neutron rich fission fragments. The beams formed in this way are not likely to be very pure, but they could be useful in a number of experiments. However, this extended project would require the installation of a low- $\beta$ initial acceleration stage and an ECR source at the LINAC, and possibly even the addition of more resonators to increase the energy of the primary beam. Recent developments of ECR ion sources allow the production of stable beams of very high intensities, which compensates the low production rate due to the thin primary target in the in-flight method. The increase of a few hundreds of Watts in the LINAC primary beam seems to be possible and would increase considerably the range of secondary beams that can be produced in RIBRAS.

A comparison between RIBRAS and other upgrade projects is shown in Fig. 3. 


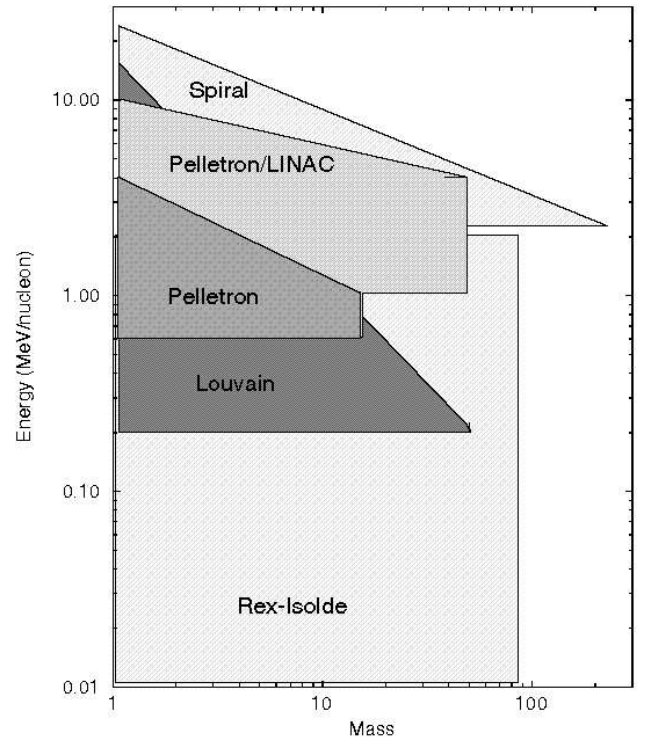

Figure 3. The regions in terms of mass and energy of operations of several RIB facilities.

\section{Recent Developments}

The two 6.5 Tesla solenoids are already mounted on the $7.5 \mathrm{~m}$ length aluminum support in the $45 \mathrm{~B}$ beam line of the Pelletron Laboratory. Both solenoids were bench tested successfully on April, 2002. The magnets were cooled down to liquid helium temperature $4 \mathrm{~K}$ and charged up to currents of 80 Amperes (magnet 2) and 50 Amperes (magnet 1). The components of the beam line for the two solenoids including primary target, Faraday cup and mid scattering ISO chambers are in the stage of installation. The Faraday cup and primary target are being manufactured. The secondary scattering chamber is being projected and will probably be man- ufactured at the Laboratório Nacional de Luz Syncrotron (LNLS), Campinas, São Paulo, Brazil.

\section{Conclusions}

A double superconducting 6.5T(5T.m) solenoid system is being installed at the Pelletron-LINAC Laboratory of the University of São Paulo in order to produce secondary beams of radioactive nuclei. The two solenoids are mounted and tested on the 45B beam line of the Pelletron experimental area. The beam line is being installed. The system shall begin its operation using the primary beams of the 8MV Pelletron Tandem and will be moved to the $10 \mathrm{MeV} /$ nucleon LINAC post-Accelerator as soon as it starts operating.

\section{References}

[1] "Proposal for the Ribras project", M.S. Hussein, A. Lépine-Szily, R. Lichtenthäler Filho, A.C.C. Villari, and V. Guimarães, (1997); Nuclear Physics Department, Annual Report 1998.

[2] "A Radioactive Beam Facility using a Large Superconducting Solenoid", J. J. Kolata, F. D. Becchetti, W. Z. Liu, D. A. Roberts, and J. W. Janecke, Nucl. Instrum. Meth. B40/41, 503 (1989).

[3] F.D. Bechetti, J.A. Brown, K. Ashktorab, J.W. Jänecke, W.Z. Liu, D.A. Roberts, R.J. Smith, J.J. Kolata, K. amkin, A. Morsad, and R.E. Warner Nucl. Instrum. and Methods in Phys. Res. B56/57, 554 (1991).

[4] S. Kubono; Nucl. Phys. A693, 221 (2001).

[5] P.D. Zecher, A. Galonsky, S.J. Gaff, J.J. Kruse, G. Kunde, E. Tryggestad, J. Wang, R.E Warner, D.J. Morrissey, K. Ieki, Y. Iwata, F. Deák, Á. Horváth, A. Kiss, Z. Seres, J.J. Kolata, J. von Schwarzenberg, and H. Schelin Phys. ReV.C57, 959 (1998).

[6] F.D. Bechetti, J.A. Brown, K. Ashktorab, J.W. Jänecke, W.Z. Liu, D.A. Roberts, R.J. Smith, J.J. Kolata, K. amkin, A. Morsad, and R.E. Warner Nucl. Instrum. and Methods in Phys. Res. B56/57, 554 (1991). 